\title{
Numerical study on the sedimentation of single and multiple slippery \\ particles in a Newtonian fluid
}

\author{
Shi Tao ${ }^{1}$, Zhaoli Guo ${ }^{1,2, *}$, Lian-Ping Wang ${ }^{1,3}$ \\ ${ }^{1}$ State Key Laboratory of coal Combustion, School of Energy and Power Engineering, Huazhong University of \\ Science and Technology, Wuhan 430074, Hubei, China \\ ${ }^{2}$ Beijing Computational Science Research Center, Beijing 100084, China \\ ${ }^{3}$ Department of Mechanical Engineering, University of Delaware, Newark, DE, 19716-3140, USA
}

\begin{abstract}
The dynamics of a single or a group of slippery spheres settling under gravity in a Newtonian fluid is studied numerically. We focus particularly on the effect of particle surface slip on the sedimentation behavior. The flows containing moving slippery spheres are solved by a three-dimensional lattice Boltzmann model where a kinetic boundary condition is used to handle the slip phenomenon at the curved particle surface. The method is first validated by simulating the slip flow in a cylindrical tube, and the no-slip flows around one and two spheres settling in a container. The hydrodynamic behaviors of one, two and multiple slippery spheres settling under gravity are then investigated. The results for a single sphere show that the surface slip makes the sphere fall faster than a no-slip particle and the wall correction factor decreases as the level of particle-surface slip is increased, indicating a drag reduction caused by the slip condition. For two settling spheres, when the no-slip particle is placed below the slip one, the two spheres will enter into the kissing phase earlier; on the contrary, deploying the no-slip particle above the slip one, the DKT process does not occur beyond a critical slip level and initial gap distance. If the two spheres are both slippery, the settling dynamics are similar to the no-slip case, but the time duration of the kissing phase decreases. As for the sedimentation of multiple spheres, it is found that the initial geometric arrangement has a significant impact on the sedimentation behavior. In general, slippery spheres in a cluster will experience larger fluctuations in the vertical velocity and position in the accelerated-falling stage, and smaller fluctuations in the decelerated-falling stage.
\end{abstract}

Keywords: Particle sedimentation; Slippery surface; Drag reduction; Lattice Boltzmann method

\section{Introduction}

The transport of particles suspended in a viscous fluid plays an important role in many natural and industrial processes involving multiphase flows, such as paper manufacturing, bed fluidization and contaminant filtration. In order to better design and control such complex particulate flow systems, it is necessary to understand fully the underlying dynamics of the particle-fluid and the particle-particle interactions. Accurate prediction of the dynamic behavior of the freely moving particles within a flow is very important and essential for such purpose, which could be obtained by the particle-resolved direct numerical simulation (PR-DNS) [1]. For PR-DNS, there are mainly two strategies available in the literature. One is the arbitrary Lagrange-Euler (ALE) method which uses a body-fitted grid and regenerates the computation mesh once any particle moves (i.e., moving mesh) [2]. Although the accuracy of boundary treatment can be guaranteed in the ALE method, the computational cost is 
significant due to grid regeneration in each time step. This problem becomes more serious when dealing with multiple particles. The second type adopts a fixed Cartesian grid over which particle-fluid interfaces move, such as the immersed boundary method (IBM) $[3,60]$, the fictitious domain method (FDM) [4], and the lattice Boltzmann method (LBM)[5]. Since no re-meshing is needed, the second approach is computationally more efficient and makes the investigation of large number of particles possible. Hence, the second approach becomes more and more widely-used in simulating particulate flows. Particularly, LBM is employed in the present study.

LBM is an alternative computational approach for solving the Navier-Stokes equations [6,7]. Pioneered by Ladd et al. [8,9] and Aidun et al. [10] in 1990s, it has now been developed into a popular numerical tool for particulate flows [11-13]. As a special discretization of the Boltzmann equation on regular Cartesian grids with minimal discrete velocity sets, the fluid dynamics in LBM is described by streaming and collision procedures of the discrete distribution functions. The no-slip boundary condition at the fluid-solid interface can be realized by the bounce-back (BB) rule, while the hydrodynamic force can be directly obtained using the momentum exchange (ME) scheme. In the original methods [8-10], the geometric shape of moving particle is approximated as low-order zig-zag lines (see Fig. 1). This is improved subsequently by the introduction of curved boundary conditions $[7,14]$ that can well preserve the geometric integrity of particle surface. Some further improvements have been proposed in recent years. For example, Wang et al. [15] substituted the multiple- relaxationtime (MRT) collision model for the single-relaxation-time (SRT) model to enhance the numerical stability. Lorenz et al. [16] proposed a corrected ME scheme to fulfill the Galilean invariance in force calculation, and the IBM and FDM have also been combined with the LBM to make use of their individual advantages $[17,18]$.

The underlying dynamics of particle-fluid and particle-particle interactions are important issues in particulate flows, and have gained much attention in the past decades. For instance, Cate et al. [19] investigated a sphere settling under gravity in a closed channel numerically, and their results agree well with experimental data for the particle Reynolds number (Re) ranging from 1.5 to 31.9. Horowitz et al. [20] studied experimentally the effect of Re on the dynamics and wake of a falling sphere, in which oscillatory motion was found and several nontrivial vortex-shedding modes were reported, and an extended parametric study was carried out by Zhou et al. [21]. Ern et al. [22] then presented a comprehensive review on the dynamics of a body falling in fluids. The case of two settling particles is also frequently studied in the literature. Fortes et al. [23] first observed experimentally that two identical spheres settling in a vertical channel would undergo drafting, kissing and tumbling (DKT) motions, which was later confirmed and reproduced in many works [24,25,57]. Recently, Wang et al. [58] and Liao et al. [26] both found that the diameter ratio of the two spheres had significant influence on such dynamic process. In particular, the occurrence of kissing could not be observed for the diameter ratio larger than 2.2, when the smaller sphere is placed above [26]. As for multiple particles, Ernst et al. [27] performed numerical simulations to investigate the settling morphology of a poly-sized sphere cluster. Nguyen et al. [28] investigated a settling suspension with focus on the influence of domain boundary at low flow Reynolds numbers. More recently, Uhlmann et al. [29] emphasized on the effect of clustering on the settling of spheres in periodic domain at moderate Reynolds numbers.

All the aforementioned studies concerned the no-slip particles, i.e., assuming that the fluid and solid share the same velocity at the solid-fluid interface. Such boundary condition (BC) can be violated in some circumstances, such as the self-cleaning lotus leaf and cicada wing, and other artificial superhydrophobic surface [30]. Apparent slip can also be found at porous surface [31] and channel flow with 
effect of electric double layer [59]. Moreover, gas flow generates a slip velocity in some systems with characteristic dimension at the order of micrometers or smaller [32]. The slip effect can be generally modeled by the Navier slip BC (see Eq. (10)) [33] characterized by the slip length $l_{s}$, which is strongly influenced by the physical properties of both fluid and solid. Actually, the effect of slip on the drag reduction of a sphere had long been noticed ever since Basset [34] where a classic drag-correlation was derived by solving the Stokes equation coupled with the Navier slip BC. The work was advanced subsequently to further consider the effects of particle-fluid viscosity ratio [35], finite Reynolds number [36], the presence of slip or no-slip walls [37] and even other particles [38]. Recently, the slip effect has been used to optimally control the wake of cylinder by carefully designing the distribution of slip region on the surface [39,40]. Daniello et al. [41] further investigated the vortex-induced vibration (VIV) of a super-hydrophobic cylinder, while Van et al. [42] considered the VIV of a three dimensional cylinder with one end fixed at the wall in a slip flow.

The dynamics of slippery particles suspended freely in Newtonian fluids had also been reported for vanishing Re in some recent works. Mandal et al. [43] studied the cross-streamline migration (CSM) of a slippery drop in an unbounded Poiseulle flow, and found a nontrivial phenomenon that the particle with significant interface slippage always moved to the centerline of channel. The interception of two slippery spheres in a linear Stokes flow was investigated by Luo et al. [44]. A critical slippage was suggested below which the surface of two particles collided after a finite time. Some works have also been reported on the suspensions of Janus particles, where half of the surface obeys the no-slip and the other half obeys the slip BC, also known as the stick-slip BCs. For example, Sun et al. [45] studied the Stokesian dynamics of pill-shaped Janus particles, and quantified the strength of the force and torque experienced by such particles. Ramachandran et al. [46] investigated analytically the dynamics of a Janus sphere in a linear flow. It was found that the stick-slip sphere undergoes a Jeffery orbit. Most recently, Trofa et al. [47] exhibited the CSM behavior of a slippery sphere suspended in a channel. The non-Newtonian effect of fluid was considered, under the Stokes flow conditions.

As outlined above, most works in the literature for suspension flow of slippery particles are limited to the Stokes regime, and the subject of a slippery particle settling under gravity at finite Re numbers has not been well studied, and even fewer works consider the effect of slip on the sedimentation dynamics of particle clusters. In this study, we will present some direct numerical simulations of the settling behaviors of one, two and multiple slippery spheres, via the lattice Boltzmann method coupled with the Navier slip boundary condition. The main object of the present work is to quantify the effect of particle surface slip on the dynamics of finite Re suspensions.

The remaining part of this paper is organized as follows. A brief introduction of the lattice Boltzmann method for particulate flows is provided in Section 2, which is validated in Section 3 with three test cases. In Section 4, we present the simulation results of one, two and multiple slippery sphere settling under gravity, focusing on the effect of slip on the dynamics of sedimentation. Finally, conclusions are given in Section 5.

\section{Methodology}

\subsection{Lattice Boltzmann method}

The viscous Newtonian fluid immersed with dispersed particles is governed by the Navier-Stokes (N-S) equations. There are many numerical approaches to solve the N-S equations, such as the classical CFD methods and the LBM. As mentioned previously, the LBM is adopted in the present work for its 
efficiency in computation and handling the complex boundaries. A detailed description of the method can be found in previous studies [6,7]. A brief introduction is given below.

As a kinetic method, the fluid fictitious particles in LBM are subject to the lattice Boltzmann equation

$$
f_{i}\left(\boldsymbol{x}+\boldsymbol{e}_{i} \delta t, t+\delta t\right)-f_{i}(\boldsymbol{x}, t)=\Omega_{i}(f), \quad i=0,1, \ldots, b-1,
$$

which describes the evolution of distribution function $f_{i}$ on Cartesian grids with selective velocity $\boldsymbol{e}_{i}$. $\Omega_{i}(f)$ denotes the discrete collision operator, $\delta t$ the time step and $b$ the total number of discrete velocities. The most widely used collision operator in LBM is the Bhatnagar-Gross-Krook (BGK) or single-relaxation-time model. However, it is pointed out in some studies that the lattice BGK may suffer from unphysical numerical artifacts at solid boundary and numerical instability [48]. To avoid the problem, we choose the so-called multi-relaxation-time (MRT) model [6] in this work, which is given by

$$
\Omega_{i}(f)=-\sum_{j}\left(\boldsymbol{M}^{-1} \boldsymbol{S} \boldsymbol{M}\right)_{i j}\left(f_{j}-f_{j}^{e q}\right),
$$

where $\boldsymbol{M}$ is a $b \times b$ transform matrix, and $\boldsymbol{S}$ is a diagonal relaxation matrix; $f_{j}^{e q}$ is the equilibrium distribution function which depends on the density $\rho$, velocity $\boldsymbol{u}$, and temperature $T$ of the gas and is typically defined as [49]

$$
f_{j}^{e q}=\omega_{j} \rho\left(1+\frac{\boldsymbol{e}_{j} \cdot \boldsymbol{u}}{c_{s}^{2}}+\frac{\left(\boldsymbol{e}_{j} \cdot \boldsymbol{u}\right)^{2}}{2 c_{s}^{4}}-\frac{u^{2}}{2 c_{s}^{2}}\right), \quad j=0,1, \ldots, b-1,
$$

where $\omega_{j}$ is the model-dependent weight coefficient, $c_{s}=\sqrt{R T}$ ( $R$ is the gas constant) the lattice sound speed. For isothermal flows, $c_{s}$ is set to be $c / \sqrt{3}$ with $c=\delta_{x} / \delta_{t}$, where $\delta_{x}$ is the lattice spacing $(c=1$ in this paper). Through the Chapman-Enskog expansion, the macroscopic fluid density $\rho$, and velocity $\boldsymbol{u}$, can be derived as the zeroth and first order moments of $f_{i}$ respectively,

$$
\rho=\sum_{i=0}^{b-1} f_{i}, \quad \rho \boldsymbol{u}=\sum_{i=0}^{b-1} \boldsymbol{e}_{i} f_{i} .
$$

The fluid pressure is defined directly as $p=c_{s}^{2} \rho$, and the viscosity of fluid is related to the relaxation time $\tau_{s}$ for the shear moment (defined in Eq. (9)) as

$$
\mu=\rho c_{s}^{2}\left(\tau_{s}-1 / 2\right) \delta t
$$

For simplicity and without loss of generality, the D3Q19 model (three dimensions with nineteen lattice velocities) is employed in this study [49], in which the velocity set and the corresponding weight coefficients are defined as

$$
\begin{gathered}
\boldsymbol{e}_{i}=\left[\begin{array}{ccccccccccccccccccc}
0 & 1 & -1 & 0 & 0 & 0 & 0 & 1 & -1 & 1 & -1 & 1 & -1 & 1 & -1 & 0 & 0 & 0 & 0 \\
0 & 0 & 0 & 1 & -1 & 0 & 0 & 1 & 1 & -1 & -1 & 0 & 0 & 0 & 0 & 1 & -1 & 1 & -1 \\
0 & 0 & 0 & 0 & 0 & 1 & -1 & 0 & 0 & 0 & 0 & 1 & 1 & -1 & -1 & 1 & 1 & -1 & -1
\end{array}\right], \\
\omega_{i}=\left\{\begin{array}{lll}
1 / 3, & i=0 \\
1 / 18, & i=1,2, \ldots, 6 . \\
1 / 36, & i=7,8, \ldots, 18
\end{array}\right.
\end{gathered}
$$

The transform matrix $\boldsymbol{M}$ is given by [6] 


$$
\boldsymbol{M}=\left(\begin{array}{ccccccccccccccccccc}
1 & 1 & 1 & 1 & 1 & 1 & 1 & 1 & 1 & 1 & 1 & 1 & 1 & 1 & 1 & 1 & 1 & 1 & 1 \\
-30 & -11 & -11 & -11 & -11 & -11 & -11 & 8 & 8 & 8 & 8 & 8 & 8 & 8 & 8 & 8 & 8 & 8 & 8 \\
12 & -4 & -4 & -4 & -4 & -4 & -4 & 1 & 1 & 1 & 1 & 1 & 1 & 1 & 1 & 1 & 1 & 1 & 1 \\
0 & 1 & -1 & 0 & 0 & 0 & 0 & 1 & -1 & 1 & -1 & 1 & -1 & 1 & -1 & 0 & 0 & 0 & 0 \\
0 & -4 & 4 & 0 & 0 & 0 & 0 & 1 & -1 & 1 & -1 & 1 & -1 & 1 & -1 & 0 & 0 & 0 & 0 \\
0 & 0 & 0 & 1 & -1 & 0 & 0 & 1 & 1 & -1 & -1 & 0 & 0 & 0 & 0 & 1 & -1 & 1 & -1 \\
0 & 0 & 0 & -4 & 4 & 0 & 0 & 1 & 1 & -1 & -1 & 0 & 0 & 0 & 0 & 1 & -1 & 1 & -1 \\
0 & 0 & 0 & 0 & 0 & 1 & -1 & 0 & 0 & 0 & 0 & 1 & 1 & -1 & -1 & 1 & 1 & -1 & -1 \\
0 & 0 & 0 & 0 & 0 & -4 & 4 & 0 & 0 & 0 & 0 & 1 & 1 & -1 & -1 & 1 & 1 & -1 & -1 \\
0 & 2 & 2 & -1 & -1 & -1 & -1 & 1 & 1 & 1 & 1 & 1 & 1 & 1 & 1 & -2 & -2 & -2 & -2 \\
0 & -4 & -4 & 2 & 2 & 2 & 2 & 1 & 1 & 1 & 1 & 1 & 1 & 1 & 1 & -2 & -2 & -2 & -2 \\
0 & 0 & 0 & 1 & 1 & -1 & -1 & 1 & 1 & 1 & 1 & -1 & -1 & -1 & -1 & 0 & 0 & 0 & 0 \\
0 & 0 & 0 & -2 & -2 & 2 & 2 & 1 & 1 & 1 & 1 & -1 & -1 & -1 & -1 & 0 & 0 & 0 & 0 \\
0 & 0 & 0 & 0 & 0 & 0 & 0 & 1 & -1 & 1 & -1 & 0 & 0 & 0 & 0 & 0 & 0 & 0 & 0 \\
0 & 0 & 0 & 0 & 0 & 0 & 0 & 0 & 0 & 0 & 0 & 0 & 0 & 0 & 0 & 1 & -1 & 1 & -1 \\
0 & 0 & 0 & 0 & 0 & 0 & 0 & 0 & 0 & 0 & 0 & 1 & -1 & -1 & 1 & 0 & 0 & 0 & 0 \\
0 & 0 & 0 & 0 & 0 & 0 & 0 & 1 & -1 & 1 & -1 & -1 & 1 & -1 & 1 & 0 & 0 & 0 & 0 \\
0 & 0 & 0 & 0 & 0 & 0 & 0 & -1 & -1 & 1 & 1 & 0 & 0 & 0 & 0 & 1 & -1 & 1 & -1 \\
0 & 0 & 0 & 0 & 0 & 0 & 0 & 0 & 0 & 0 & 0 & 1 & 1 & -1 & -1 & -1 & -1 & 1 & 1
\end{array}\right),
$$

and the relaxation matrix is

$$
S=\operatorname{diag}\left(\tau_{\rho}, \tau_{e}, \tau_{\varepsilon}, \tau_{d}, \tau_{q}, \tau_{d}, \tau_{q}, \tau_{d}, \tau_{q}, \tau_{s}, \tau_{\pi}, \tau_{s}, \tau_{\pi}, \tau_{s}, \tau_{s}, \tau_{s}, \tau_{t}, \tau_{t}, \tau_{t}\right)^{-1}
$$

where $\tau_{\rho}$ and $\tau_{d}$ are the relaxation times for conserved moments and can take arbitrary values. The other relaxation times are related to the non-conserved moments and their values are greater than 0.5 .

\subsection{Kinetic boundary condition for slippery particle}

In this work, the unified interpolated bounce-back scheme [14] is applied to the curved boundary of no-slip particle. As for the slippery particle, it becomes an important issue for LBM to specify a suitable boundary condition. Although the bounce-back (BB) or interpolated BB method is widely used to realize the no-slip $\mathrm{BC}$, it does not work for a slip wall.

For the slip BC, the macroscopic Navier-type scheme [33] has been widely used and is adopted in this study, which is given by

$$
u_{s}=l_{s} \partial_{n} u_{\tau}
$$

where $\boldsymbol{n}$ is the unit vector normal to the wall, and $l_{s}$ is the slip length which is strongly influenced by the physical properties of both fluid and particle, $u_{s}$ and $u_{\tau}$ are the slip and tangential velocities. It is known that the slip BC given by Eq. (10) cannot be directly implemented in LBM. Several types of kinetic schemes [7,50] have been proposed to realize the slip BC on flat walls. However, most of those available BCs are not suitable for flows in the present study, where the curved geometry of spherical wall is considered. On the other hand, the curved slip BC based on the diffusive bounce-back method (DBB, i.e., a combination of the diffuse reflection scheme and the bounce-back rule), which has good performance in the simulations of slip flow with complex boundaries [51], can serve such purpose and therefore is adopted in the present study. In the curved DBB scheme, the unknown distribution function at the boundary node, such as $B$ in Fig. 1 is specified as

$$
f_{i}=r f_{i}^{+}+(1-r) f_{i}^{e q},
$$

where the parameter $r$ denotes the portion of bounce-back part, $\bar{i}$ is the opposite direction of $i, f_{i}^{+}$is the post-collision distribution function and $f_{i}^{e q}$ the equilibrium distribution function from the wall. In 
order to retain the integrity of the wall boundary, the value of $r$ should be chosen as [51]

$$
r=\frac{\tau_{s}+q-1-N \mathrm{Kn}}{\tau_{s}-q+N \mathrm{Kn}},
$$

Here, $(1-q)$ represents the relative distance from the boundary point to the solid wall, $N$ denotes the number of lattices along the characteristic length $D$ and $\mathrm{Kn}=l_{s} / D$ is the dimensionless slip length.

It should be noted that, in the original scheme [51], the slip effect is generated from the rarefaction of fluid, where the relaxation time $\tau_{s}$ is determined by $\mathrm{Kn}$ (defined by $\lambda / D$ with $\lambda$ the molecular mean free path of gas) under the consistency requirement as [7]

$$
\tau_{s}=\frac{1}{2}+N \mathrm{Kn} \sqrt{\frac{6}{\pi}}
$$

However, when the working fluid is a liquid, the above $\tau_{s} \sim$ Kn relationship no longer exists, and the value of $\tau_{s}$ is obtained from Eq. (5), just the same as for continuum flows.

\subsection{Hydrodynamic and collision forces}

To update the position of a freely moving particle, the force and torque exerted on it have to be calculated first. The hydrodynamic force coming from the ambient fluid can be obtained directly using the momentum exchange (ME) method in LBM. It should be noted that there is no fictitious fluids in the solid particle. Therefore, the ME process happens only outside the particle [61], and we will adopt the corrected ME scheme [16] which is given by

$$
\boldsymbol{F}_{s}=\sum_{\boldsymbol{x}_{f}} \sum_{i} \frac{\delta_{x}^{3}}{\delta_{t}}\left[-\left(\boldsymbol{e}_{i} f_{i}\left(\boldsymbol{x}_{b}\right)-\boldsymbol{e}_{\bar{i}} f_{\bar{i}}^{*}\left(\boldsymbol{x}_{b}\right)\right)+\Delta M\right], \quad \Delta M=-\frac{\omega_{i} \rho}{c_{s}^{2}}\left(\frac{\left(\boldsymbol{e}_{i} \cdot \boldsymbol{u}_{w}\right)^{2}}{c_{s}^{2}}-\boldsymbol{u}_{w}^{2}\right) \boldsymbol{e}_{\bar{i}}
$$

where $x_{b}$ is the boundary node, $\boldsymbol{u}_{w}$ is the particle velocity at the point of intersection between the link and the particle surface, and $\Delta M$ is included to fulfill the Galilean invariance [16]. The torque can then be determined similarly. For the case of multiple particles confined in a container, the collisions of particle-particle and particle-wall may be inevitable. There are mainly two classes of methods to model such interactions in the literature, i.e., the discrete element method [52] and the repulsive force model [17]. The latter scheme will be used in the present study, where the collision force is given by

$$
\boldsymbol{F}_{c}= \begin{cases}0, & \left\|\boldsymbol{x}_{i}-\boldsymbol{x}_{j}\right\|>R_{i}+R_{j}+\zeta, \\ \frac{c_{i j}}{\varepsilon_{c}}\left(\frac{\left\|\boldsymbol{x}_{i}-\boldsymbol{x}_{j}\right\|-R_{i}-R_{j}-\zeta}{\zeta}\right)^{2}\left(\frac{\boldsymbol{x}_{i}-\boldsymbol{x}_{j}}{\left\|\boldsymbol{x}_{i}-\boldsymbol{x}_{j}\right\|}\right), & \left\|\boldsymbol{x}_{i}-\boldsymbol{x}_{j}\right\| \leq R_{i}+R_{j}+\zeta .\end{cases}
$$

Here, $c_{i j}$ is the force scale defined as the buoyancy force in the suspension flows; $\varepsilon_{c}$ is the stiffness parameter for collisions and is set to be $0.01 ; R_{i}$ and $R_{j}$ are the radii, and $\boldsymbol{x}_{i}$ and $\boldsymbol{x}_{j}$ are the corresponding centers of two particles; $\zeta$ is the threshold gap distance and takes a value of $1.5 \delta_{x}$ in this paper. As for particle-wall collision, $\boldsymbol{x}_{j}$ is the position of a fictitious particle which is located symmetrically on the other side of the wall and $R_{j}=R_{i}$. It is worth mentioning that the collision force always points to the center of sphere and hence it does not contribute to the torque on the particle. After the force and torque exerted on the particle are obtained, the trajectory can then be tracked by the Newton's second law,

$$
M_{s} \frac{d \boldsymbol{u}_{s}}{d t}=\boldsymbol{F}_{s}+\boldsymbol{F}_{e}, \boldsymbol{I}_{s} \frac{d \boldsymbol{\phi}_{s}}{d t}=\boldsymbol{T}_{s}
$$

where $M_{s}$ and $\boldsymbol{I}_{s}$ are the mass and the inertia moment of particle, respectively; $\boldsymbol{u}_{s}$ and $\boldsymbol{\phi}_{s}$ are the translational velocity and the rotational velocity of particle $\left(\boldsymbol{u}_{w}=\boldsymbol{u}_{s}+\boldsymbol{\phi}_{s} \times\left(\boldsymbol{x}_{w}-\boldsymbol{x}_{c}\right), \boldsymbol{x}_{w}\right.$ and $\boldsymbol{x}_{c}$ are the positions of intersecting point and particle center respectively); $\boldsymbol{F}_{s}$ and $\boldsymbol{T}_{s}$ are the force and the torque 
coming from the surrounding fluid, and $\boldsymbol{F}_{e}$ is other external force, such as the body force or the collision force. The translation and rotation velocities of the particle are updated by solving Eq. (16) with the first-order Euler method,

$$
\boldsymbol{u}_{s}^{n+1}=\boldsymbol{u}_{s}^{n}+\delta_{t}\left(\boldsymbol{F}_{s}+\boldsymbol{F}_{e}\right) / M_{s}, \quad \boldsymbol{\phi}_{s}^{n+1}=\boldsymbol{\phi}_{s}^{n}+\delta_{t} \boldsymbol{T}_{s} / \boldsymbol{I}_{s},
$$

The particle position $\boldsymbol{x}_{s}$ and rotation angle $\theta$ can then be obtained as

$$
\boldsymbol{x}_{s}^{n+1}=\boldsymbol{x}_{s}^{n}+\boldsymbol{u}_{s}^{n} \delta_{t}+\frac{1}{2} \delta_{t}^{2}\left(\boldsymbol{F}_{s}+\boldsymbol{F}_{e}\right) / M_{s}, \quad \theta_{s}^{n+1}=\theta_{s}^{n}+\phi_{s}^{n} \delta_{t}+\frac{1}{2} \delta_{t}^{2} \boldsymbol{T}_{s} / \boldsymbol{I}_{s},
$$

\section{Validation}

In this section, we validate the LBM by considering three test cases: the slip flow in a long cylindrical channel, the sedimentation of a sphere in a closed box, and the DKT process of two spheres settling in a container. The reliability is confirmed by comparing the results with the available experimental and numerical data in the literature. The relaxation times are chosen as $\tau_{e}=1 / 1.19, \tau_{\varepsilon}=\tau_{\pi}$ $=1 / 1.14, \tau_{q}=\left(8 \tau_{s}-1\right) /\left(16 \tau_{s}-8\right)$ and $\tau_{t}=1 / 1.98$ following Refs. [6,50], which are found to have negligible influence on the simulation results.

\subsection{Slip flow in a tube}

The first test problem is the slip flow in a cylindrical tube. It is a classical problem and has an analytical solution [53]. The steady-state flow is driven by a pressure gradient $\partial p / \partial x$ in a tube with radius $R$, and governed by the reduced N-S equation which can be expressed in the cylindrical coordinate as

$$
\frac{1}{r} \cdot \frac{\partial}{\partial r}\left(r \frac{\partial u}{\partial r}\right)=\frac{1}{\mu} \cdot \frac{\partial p}{\partial x}
$$

where $u$ is the streamwise velocity and $r$ is the radial distance. The slip and symmetry boundary conditions are given by

$$
u_{s}=\left.l_{s} \frac{\partial u}{\partial r}\right|_{r=R},\left.\quad \frac{\partial u}{\partial r}\right|_{r=0}=0 .
$$

The velocity profile can be obtained as

$$
u(r)=\frac{1}{4 \mu} \frac{\partial p}{\partial x}\left[r^{2}-R^{2}(1+4 \mathrm{Kn})\right], \quad r=0 \sim R .
$$

with $\mathrm{Kn}=l_{s} /(2 R)$.

In the simulations, the pressure gradient is realized by specifying different fluid densities at the tube inlet and outlet, with $\rho_{\text {in }}=1.0001$ and $\rho_{\text {out }}=0.9999$ respectively. The pressure boundary conditions are then realized by the non-equilibrium extrapolation method [62]. The curved DBB scheme, i.e., Eq. (11) is implemented to exert the slip BC at the cylindrical wall of the tube. The ratio of tube length to diameter is $10: 1$, and the grid spacing is $D / 16$, where $D=2 R$ is the tube diameter. It is found that the length of the tube is long enough to eliminate the entrance effect. Fig. 2 shows the velocity profiles of the flow at $\mathrm{Kn}=0.02$ and 0.06 , with $u_{0}=-R^{2}(\partial p / \partial x) / 4 \mu$ the maximum streamwise velocity of no-slip flow. As can be seen, the results are in good agreement with the analytical solution of the N-S equation.

\subsection{Sedimentation of a sphere in a closed box}


A sphere settling in a closed rectangular cavity is investigated as the second test case, for which experimental and numerical data are available in the literature $[19,26]$. The size of the closed box is 10 $\times 16 \times 10 \mathrm{~cm}^{3}$, and is filled with a viscous Newtonian fluid. Four sets of density and dynamic viscosity are considered, namely, $\left(\rho_{f}, \mu\right)=\left(0.97 \mathrm{~g} / \mathrm{cm}^{3}, 3.73 \mathrm{~g} / \mathrm{cm} \cdot \mathrm{s}\right),\left(0.965 \mathrm{~g} / \mathrm{cm}^{3}, 2.12 \mathrm{~g} / \mathrm{cm} \cdot \mathrm{s}\right),\left(0.962 \mathrm{~g} / \mathrm{cm}^{3}\right.$, $1.13 \mathrm{~g} / \mathrm{cm} \cdot \mathrm{s})$ and $\left(0.96 \mathrm{~g} / \mathrm{cm}^{3}, 0.58 \mathrm{~g} / \mathrm{cm} \cdot \mathrm{s}\right)$, respectively. A sphere with diameter $D=1.5 \mathrm{~cm}$ and density $\rho_{p}=1.12 \mathrm{~g} / \mathrm{cm}^{3}$ starts to fall under gravity $\left(g=980 \mathrm{~cm} / \mathrm{s}^{2}\right)$ at a gap distance of $H_{p}=12 \mathrm{~cm}$ from the sphere to the bottom wall of channel. The corresponding particle Reynolds numbers $\left(\operatorname{Re}=V D \rho_{f} / \mu\right.$ with $V$ the terminal velocity of the settling sphere) are 1.5, 4.1, 11.6 and 31.9, which have been reported in Ref. [19] from the experimental measurements using the particle image velocity (PIV) technique.

In analogy to the experiments [19], the present simulations are performed in a vertical channel of size $120 \times 192 \times 120$ in lattice units. This implies that the sphere is resolved by 18 lattices, and initially located at a height of 153 lattices. The boundaries of channel in all the three directions are static walls, and realized by a non-equilibrium extrapolation method in LBM [62]. The relaxation time $\tau_{s}$ is set to be 1.0. Then the acceleration of gravity is determined from dimensional analysis. The time histories of the vertical position and velocity of the particle are presented in Fig. 3. As can be seen, the sphere settles faster with increasing Re. In general, there are three regimes in the sedimentation process: acceleration, steady sedimentation and deceleration when approaching the bottom wall. The present LBM have reproduced these stages of sedimentation, as shown in Fig. 3(b). The results agree well with the experimental data [19] and the numerical results [26].

\subsection{DKT process of two settling spheres}

In particulate flows, a particle not only interacts with the fluid, but also undergoes inter-particle interactions in the presence of other particles. Therefore, the simulation of a particle pair settling under gravity is performed to further evaluate the LBM in modeling multiple particle systems. This standard test case has been investigated by several authors. In the present study, the parameters are chosen from Refs. $[24,25,54]$. The domain of the container is $[0,6 D] \times[-6 D, 24 D] \times[0,6 D]$ with $D=1 / 6 \mathrm{~cm}$ being the sphere diameter. The fluid density and dynamic viscosity are respectively $\rho_{f}=1.0 \mathrm{~g} / \mathrm{cm}^{3}$ and $\mu=$ $0.01 \mathrm{~g} / \mathrm{cm}$.s. The upper and lower spheres are identical with a density of $\rho_{p}=1.14 \mathrm{~g} / \mathrm{cm}^{3}$. The initial positions of the two spheres are $(3.03 D, 21 D, 3.03 D)$ and $(2.97 D, 18.96 D, 2.97 D)$, respectively. No-slip boundary conditions are applied at all the domain boundaries [62]. The no-slip BC at the moving particles is realized by a unified interpolated bounce-back scheme [14]. In the simulations, the sphere is resolved by 14 lattices, which corresponds to a lattice system of $84 \times 420 \times 84$ for the computational domain.

Figure 4 presents the instantaneous positions of the two spheres during the settling process. The DKT phenomenon is clearly reproduced. Initially, the two spheres are located along the centerline of channel with a relatively small gap. After released from rest in the still fluid, both particles begin to descend under gravity. While the leading sphere is falling down, it creates a wake with lower pressure. As the trailing particle comes close to the leading one, it is drafted into the wake and experiences a much smaller drag. Hence, the trailing sphere moves faster than the leading one, and eventually catches up, and then kisses and impels the latter. This stage persists for several periods, during which the spheres form a doublet and fall downwards together. However, that state is unstable as indicated in Refs. [23,27], because of some symmetry breakings such as the fluctuating wake. As a result, the sedimentation process turns into the tumbling stage, where the particles start to separate from each 
other. The time histories of the distance between the spheres and the vertical velocities of the particles are given in Fig. 5. It can be seen that after about $0.15 \mathrm{~s}$, the settling velocity of the trailing sphere increases faster and exceeds that of the leading one (Fig. 5(b)), and the gap decreases dramatically (Fig. 5(a)). At about $t=0.33 \mathrm{~s}$, the distance approaches to a local minimum value (Fig. 5(a)), indicating a contact with each other, and this kissing stage lasts about $0.21 \mathrm{~s}$. Finally, at about $t=0.54 \mathrm{~s}$, the distance increases and the particles start to separate. As shown in Fig. 5, the DKT processes predicted by the LBM agree well with those reported by Yang et al. [25] using the immersed boundary method and Apte et al. [24] using the fictitious domain method. However, the tumbling stages show significantly differences among the different simulations. As indicated in Refs. [23,27], the difference can be expected in that the implementation of tumbling relies heavily on the growth rate of the numerical uncertainties and the boundary treatments as well as the collision models. Therefore, the present LBM model can be generally considered to be able to give reasonable results for the DKT dynamics of two no-slip spheres.

\section{Results and discussions of sedimentation of slippery spheres}

In this section, the simulations of one, two and multiple slippery spheres settling under gravity in a channel with a square cross-section are performed respectively to investigate the effect of particle surface slip on the sedimentation dynamics. When the simulations involve more than one particle, the influences of the number of slippery particles and the initial geometric arrangement are also investigated. For all cases considered below, the fluid density, viscosity and the sphere diameter are set to $\rho_{f}=1.0 \mathrm{~g} / \mathrm{cm}^{3}, \mu=0.01 \mathrm{~g} / \mathrm{cm} . \mathrm{s}$ and $D=0.08 \mathrm{~cm}$, respectively. The particle density $\rho_{p}$ and channel width $W$ are adjustable, which provide two control parameters, i.e., the density ratio $\rho_{r}=\rho_{p} / \rho_{f}$ and the blockage ratio $W^{*}=W / D$. The channel length is $H$, and the Knudsen number defined by $l_{s} / D$ is less than 0.12 in the simulations.

\subsection{Single slippery sphere settling in a narrow open channel}

The sedimentation of a slippery sphere in a narrow open channel is first investigated, with $W^{*}=4.0$ and $\rho_{r}=1.001$. In the simulations, the particle diameter takes 20 lattices and the channel has a length of $H=30 D$. The fluid at the lower end of the channel is assumed to be stationary, and free-stream boundary condition is applied to the upper end; no-slip boundary conditions are applied to the channel walls [62]. To reduce the entrance effect, the sphere always stays at the center of channel, i.e., with a distance of $15 D$ to the two vertical boundaries of channel. It is accomplished by moving the computational domain a lattice downwards once the sphere descends a lattice. Fig. 6 displays the instantaneous positions of a settling slippery sphere at $\mathrm{Kn}=0.1$, the results of a no-slip sphere are also included for comparison. It is clearly observed that the particle with surface slip falls faster than the one without slip, suggesting that the resistance experienced by the slippery particle is reduced. Actually, the terminal settling velocities are $0.0234 \mathrm{~cm} / \mathrm{s}$ and $0.0191 \mathrm{~cm} / \mathrm{s}$, for the slip and no-slip cases, respectively. It can be found that in both cases the particle Reynolds number is less than 0.2.

For the sedimentation of a particle in a relatively narrow channel, it is important to investigate the effect of channel blockage on the settling process. This influence is generally described quantitatively by the so-called wall correction factor $K$, which is defined as

$$
K=\frac{C_{d}}{C_{d_{0}}}, \quad C_{d}=\frac{F_{d}}{3 \pi \mu V D}, \quad C_{d_{0}}=\frac{1+4 \mathrm{Kn}}{1+6 \mathrm{Kn}} .
$$


Here, $F_{d}$ is the drag force and $V$ the terminal settling velocity of particle, $C_{d}$ is the drag coefficient and $C_{d_{0}}$ denotes the value of $C_{d}$ for a sphere in the unbounded Stokes flow $(\operatorname{Re}<0.2)$. The value of $C_{d_{0}}$ has been obtained analytically by many works, such as Basset et al. [55] and Feng et al. [36], where the Stokes equations coupled with the Navier slip BC over a sphere were solved. In the present simulations, $W^{*}$ ranges from 2.0 to 10.0 , and $\rho_{r}$ is adjusted to guarantee Re less than 0.2 . The predicted results of $K$ are given in Fig. 7. The correction factor for a no-slip sphere moving in a cylindrical tube along the axis reported by Happle et al. [56],

$$
K=\frac{1}{\left[\begin{array}{l}
1-2.10443\left(W^{*}\right)^{-1}+2.08877\left(W^{*}\right)^{-3}-0.94813\left(W^{*}\right)^{-5}-1.372\left(W^{*}\right)^{-6} \\
+3.87\left(W^{*}\right)^{-8}-4.19\left(W^{*}\right)^{-10}
\end{array}\right]},
$$

is also included for comparison (open symbols in Fig. 7). It is clear that the wall correction factor generally decreases with increasing $\mathrm{Kn}$. This is consistent with the findings in Fig. 6 where sphere with slip BC settles faster. This can also be explained by the fact that the effective no-slip size of the particle becomes smaller when slip occurs at the surface, compared to its real physical diameter; hence, the blockage ratio $K$ decreases. Furthermore, $K$ is a monotonic decreasing function of the blockage ratio $W^{*}$. In particular, as $W^{*}$ is larger than 4 , the effect of slip is weakened significantly. When $\mathrm{Kn}$ is close to zero, the present results can generally reduce to those obtained from Eq. (23). The deviation, particularly for smaller $W^{*}$ can also be found because Eq. (23) is for a sphere settling in a cylindrical tube, while the cross-section of channel is a square in the present work.

In order to explore the effect of slip for finite Re, simulations are also performed with a larger density ratio at $\rho_{r}=1.1$. The results are presented in Fig. 8, which shows that the particle Reynolds number, Re, defined in terms of the terminal velocity, increases nonlinearly with $\mathrm{Kn}$, and the relative increase in the settling rate from $\mathrm{Kn}=0$ to $\mathrm{Kn}=0.12$ appears to be independent of the blockage ratio.

\subsection{Sedimentation of two slippery spheres in a container}

We now investigate the effect of slip on the DKT dynamics of two spheres settling in a container with static walls. Two cases are considered here, i.e., (i) a slippery sphere and a no-slip one; and (ii) both slippery spheres. For Case-I, the slippery particle can be placed above or below the no-slip one, denoted as the slip-up and the slip-down, respectively (shown in Fig. 9(a)). In the simulations, we set $W^{*}=6.0$ and $\rho_{r}=1.05$; the channel length is $H=30 D$. The particles are released from the centerline of channel with an initial gap of $1.04 D$.

Figure 9 presents the distance between the two spheres in Case-I. It can be seen that, in the drafting phase, the particle gaps in the slip-down and slip-up setups are respectively larger and smaller than that for the case of two no-slip spheres. Hence, the occurrence of particle collision can be postponed in the former case, while accelerated in the latter case. Such results can be attributed to two factors. One is that, as outlined in Section 3.3, the trailing particle is located in the low pressure wake of the leading one, such that it experiences a lower drag and moves faster. The other is because the slip effect also contributes to a faster translation, as indicated in Section 4.1. Therefore, if the slippery particle is placed above the no-slip one, i.e., the slip-up setup, the two factors are mutually reinforced so that the gap decreases more rapidly. On the other hand, in the slip-down setup, the two factors are in competition and as such weaken each other, hence resulting in slower approaching of the two spheres. Particularly, for the slip-down setup, a short period of increasing gap is even observed clearly at $\mathrm{Kn}=$ 0.03 and 0.06 . At $\mathrm{Kn}=0.12$, the gap continues to increase as the slip effect dominates, but decreases 
abruptly at around $t=1.72 \mathrm{~s}$ due to the fact that the leading particle has already reached the bottom wall, as shown in Fig. 9(b). Hence, it can be expected that there exists a critical Knudsen number (Kn)c and gap $(\delta / D)_{c}$ for the slip-down setup, beyond which the trailing no-slip particle can never catch up with the leading slippery particle. To find out such $(\mathrm{Kn})_{c}$ and $(\delta / D)_{c}$, simulations with small increments of $\mathrm{Kn}($ at $\delta / D=1.04$ ) and $\delta / D$ (at $\mathrm{Kn}=0.06$ ) are performed, respectively, for the slip-down setup. The results are presented in Fig. 10. It is clear that the time for the occurrence of kissing will be postponed with larger Kn and initial gap. A critical Knudsen number, $(\mathrm{Kn})_{c}=0.085$, and initial gap, $(\delta / D)_{c}=1.5$, respectively, can be found under which the two spheres collide with each other just in time when the leading slippery particle reaches the bottom of container. For $\mathrm{Kn}<0.085$ and $\delta / D<1.5$, the kissing process occurs before the leading sphere reaches the bottom. No kissing is observed for $\mathrm{Kn}>0.085$ at $\delta / D=1.04$, or $\delta / D>1.5$ at $\mathrm{Kn}=0.06$.

For Case-II (both being slip particles), the time history of particle gap seems to be unaffected by Kn during the drafting stage $(t<0.64 \mathrm{~s})$ compared to the no-slip case, as shown in Fig. 11(a). Actually, from Fig. 11(b), it is clear that the vertical velocities of both spheres are larger than those for two no-slip spheres, as slippery surface contributes to a lower drag. However, the velocity augmentations of the two spheres are comparable to each other, leading to an almost unchanged gap distance. For different $\mathrm{Kn}$, the particles begin to collide all at about $t=0.64 \mathrm{~s}$, but the time period of the kissing stage is a decreasing function of $\mathrm{Kn}$, which can be qualitatively understood by the decreasing effective particle size due to slip. The tumbling stage appears earlier for higher Kn as observed in Fig. 11(a). This is reasonable since the occurrence of symmetry breaking is easier in the flow with a higher Re. At about $t=1.4 \mathrm{~s}$, the vertical velocities tend to be steady and their values are mainly determined by Kn, as shown in Fig. 11(b).

\subsection{Sedimentation of multiple slippery spheres in a closed box}

In order to further explore the effect of slip on the sedimentation dynamics of a particle cluster, we now simulate the settling process of 96 spheres in a container. The initial configuration of the particles is arranged as a $4 \times 4 \times 6$ block, as shown in Fig. 12(a). The computational parameters in the simulation are summarized as follows, $W / D=6.0, \rho_{p} / \rho_{f}=1.1$ and $\delta x=D / 16$. The length of the channel is $H=50 D$, and the degree of slip is fixed at $\mathrm{Kn}=0.1$. Three main cases are considered here: all the spheres are no-slip (no-slip); all the spheres are slip (slip); half of the spheres are slip and half no-slip, respectively. The third case is further dived into four sub-cases according to the arrangements: the slippery spheres are all placed below the no-slip spheres (no-slip + slip) or all placed above the no-slip ones (slip + no-slip), and one layer of no-slip particles and one layer of slip particles that cycles from Layer (1) to (6) (no-slip + slip + no-slip + slip), or reverse the orders (slip + no-slip + slip + no-slip). Therefore, a total number of six cases are considered here. The characteristic velocity and time are respectively defined as

$$
U=\sqrt{\frac{\pi D\left(\rho_{r}-1\right) g}{2}}, \quad T=D / U .
$$

Some other parameters that characterize the particle dynamics are the fluctuation velocity $v^{\prime}$ in the vertical direction, vertical r.m.s. dispersion $h^{\prime}$, and the collision number $C N$, which are defined as

$$
v^{\prime}=\sqrt{\frac{\sum_{i=1}^{N}\left(V_{i}-\bar{V}\right)^{2}}{N U^{2}}}, \quad h^{\prime}=\sqrt{\frac{\sum_{i=1}^{N}\left(h_{i}-\bar{h}\right)^{2}}{N D^{2}}}, \quad C N=\frac{C_{n}}{N},
$$

where $N$ is the particles number, $C_{n}$ the total collision number of a pair of particles, $V_{i}$ and $h_{i}$ are vertical 
velocity and position of the $i$-th particle, respectively, $\bar{V}$ and $\bar{h}$ are the corresponding average values.

Figure 12 presents the instantaneous vortex structures around the particles at different times. The spheres are all slippery with $\mathrm{Kn}=0.1$. As can be seen from Figs. 12(b) to 12(d), the inner spheres in the cluster fall faster than the outer ones, exhibiting unsteadiness similar to the Rayleigh-Taylor instability. After that, i.e., as $t>47.8$, the DKT dynamics and particle rearrangements occur repeatedly. The cluster continues to develop in the vertical direction. At about $t=95.5$ (Fig. 12(f)), some spheres approach to the channel bottom, and the sphere cluster starts to contract and is eventually packed at the bottom, as shown in Fig. 12(j).

Figure 13 displays the time evolutions of the vertical fluctuation velocity $v^{\prime}$ and position $h^{\prime}$, and the collision number $C N$ during the sedimentation process. It can be found in Fig. 13(a) that, despite of the different geometric arrangements, the six particle clusters considered generally undergo three stages, i.e., accelerated falling, steady falling and decelerated falling, corresponding to the time ranges $0 \leq t<$ $t_{1}, t_{1} \leq t<t_{2}$ and $t>t_{2}$, respectively. In the earlier stage, i.e., $0 \leq t<t_{1}$, the fluctuation velocities of the four cases with particles in staggered arrangements are generally larger than those in the no-slip and the slip cases. As presented in section 4.1, slip particles moves faster than the no-slip ones. Therefore, if slip and no-slip spheres are placed in a staggered fashion, larger velocity differences are generated. Particularly, the slip + no-slip case produces the greatest $v^{\prime}$ at about $t=t_{1}$. The snapshot of the velocity of each particle at the time is also presented, and the no-slip case is shown as well for comparison. From the two figures, it can be clearly observed that the particle velocities can differ significantly in the slip + no-slip case, but is relatively homogeneous in the no-slip case. For $h$ ' shown in Fig. 13(b), the value in the no-slip + slip case is generally larger than that in the slip + no-slip case, while the relationship of velocity fluctuation $v^{\prime}$ of these two cases is just the opposite, as shown in Fig. 13(a). The particle distributions at $t=25$ also show that spheres in the latter case are dispersed more homogeneously than those in the former case. This finding is consistent with that from the slip-down case presented in Fig. 9 (a) where the drafting no-slip particle cannot catch up with the leading slippery particle in the earlier stage at $\mathrm{Kn}=0.1$. Hence, the settling dynamics of the no-slip + slip cluster has some similar features with those of a no-slip sphere drafted by a slip one. As to the collision number shown in Fig. 13(c), $C N$ in the slip + no-slip case ranks the first, and the lowest $C N$ comes from the slip case. The former can be explained by that all the slippery particles are in the low pressure wake, the velocity difference between the no-slip and slip particles are further enlarged which strengthens the particle mixing, hence generates the greatest $C N$. The latter finding indicates that slippery surface contributes to a reduction of particle-particle interaction, and the particle cluster will reach to a quasi-steady state earlier.

During the steady falling stage $\left(t_{1} \leq t<t_{2}\right)$, the particle velocities tend to be stable, and therefore $v^{\prime}$ descends and tends to a relatively stable value, as shown in Fig. 13(a). Comparing the two instantaneous snapshots, $B$ and $C$ of the settling velocities of particles in the slip case, we can observe that the distribution is fairly homogeneous during the steady falling stage. From Fig. 13(b), it can be found that, the values of $h$ ' of the slip case and the no-slip case are the largest and the lowest, and are the first and last to decease in the six cases, respectively. It further confirms that the cluster of slippery particles reaches to a stable state earlier than the no-slip cluster. The values of $C N$ in this stable stage stay at constant levels for each case, and the relationships between the cases are almost the same to those in the accelerated falling stage.

As $t \geq t_{2}$, the sedimentation of the cluster comes to the final decelerated falling stage. From Fig. 13(c), it can be seen that $v^{\prime}$ and $h$ 'both start to descend, while $C N$ starts to increase, significantly for all 
the six cases. During this stage, the particles will approach to and rest on the bottom of containerin a narrow region, and consequently $v^{\prime}$ and $h$ ' both decrease rapidly with time, while $C N$ increases sharply due to the closer packing of the spheres. Particularly, it is noted that the incremental rate of $C N$ in the slip case is much more pronounced than those in the other cases, as shown in Fig. 13(c), while the $C N$ in the no-slip case increases most slowly. The above results suggest that the setup with slippery particles surrounded by no-slip ones will generally increase the interaction of particles.

\section{Conclusions}

The dynamics of slippery spheres settling in a Newtonian fluid is investigated by means of three-dimensional particle-resolved direct numerical simulations. In particular, the effect of surface slip on the sedimentation process is studied. The particle-fluid flow is solved by a lattice Boltzmann method coupled with a kinetic boundary condition for velocity slip at the curved particle surfaces. After a validation of the method, simulations of one, two and multiple slippery spheres are then carried out. Comparing with the corresponding cases where only no-slip spheres are involved, the following conclusions can be drawn.

(i) For the single sphere case, the results show that the particle with surface slip falls faster than the no-slip sphere; the wall correction factor decreases with increasing slip, particularly for narrow channels. Thus, the slip effect increases the Reynolds number of settling particles.

(ii) For the two spheres case, if the no-slip particle is placed below the slip one, the two spheres will enter the kissing phase earlier in comparison with two no-slip spheres. On the contrary, if placed in the reverse order, with the no-slip particle placed being above the slip particle, the DKT process does not always occur. The critical gap $(\delta / D)_{c}$ and slip degree $(\mathrm{Kn})_{c}$, about 1.5 (for $\mathrm{Kn}=0.06$ ) and 0.085 (for $\delta / D=1.04)$ respectively, are found, over which the no-slip particle can never collide with the slip one. When the two spheres are both slippery, the settling dynamics are similar to the no-slip case, except for the earlier occurrence of tumbling process.

(iii) As for the case of multiple spheres, it is shown that the geometric arrangement can have a significant influence on the sedimentation dynamics. Putting the slippery spheres inside the particle cluster generally increases the fluctuations in vertical velocity and position in the accelerated falling stage, and attenuates these fluctuations in the decelerated stage.

\section{Acknowledgments}

This work is subsidized by the National Natural Science Foundation of China (51390494).

\section{References}

[1] Tenneti, S., \& Subramaniam, S. (2014). Particle-resolved direct numerical simulation for gas-solid flow model development. Annual Review of Fluid Mechanics, 46, 199-230.

[2] Hu, H. H., Patankar, N. A., \& Zhu, M. Y. (2001). Direct numerical simulations of fluid-solid systems using the arbitrary Lagrangian-Eulerian technique. Journal of Computational Physics, 169(2), 427-462.

[3] Kim, Y., \& Peskin, C. S. (2016). A penalty immersed boundary method for a rigid body in fluid. Physics of Fluids, 28(3), 033603.

[4] Glowinski, R., Pan, T. W., Hesla, T. I., Joseph, D. D., \&Periaux, J. (2001). A fictitious domain approach to the 
direct numerical simulation of incompressible viscous flow past moving rigid bodies: application to particulate flow. Journal of Computational Physics, 169(2), 363-426.

[5] Ladd, A. J. (2015). Lattice-Boltzmann methods for suspensions of solid particles. Molecular Physics, 113(17-18), 2531-2537.

[6] Lallemand, P., \& Luo, L. S. (2000). Theory of the lattice Boltzmann method: Dispersion, dissipation, isotropy, Galilean invariance, and stability. Physical Review E, 61(6), 6546.

[7] Guo, Z., \& Shu, C. (2013). Lattice Boltzmann method and its applications in engineering . World Scientific.

[8] Ladd, A. J. (1994). Numerical simulations of particulate suspensions via a discretized Boltzmann equation. Part 1. Theoretical foundation. Journal of Fluid Mechanics, 271(1), 285-309.

[9] Ladd, A. J. (1994). Numerical simulations of particulate suspensions via a discretized Boltzmann equation. Part 2. Numerical results. Journal of Fluid Mechanics, 271, 311-339.

[10] Aidun, C. K., \& Lu, Y. (1995). Lattice Boltzmann simulation of solid particles suspended in fluid. Journal of Statistical Physics, 81(1-2), 49-61.

[11] Rubinstein, G. J., Derksen, J. J., \& Sundaresan, S. (2016). Lattice Boltzmann simulations of low-Reynolds-number flow past fluidized spheres: effect of Stokes number on drag force. Journal of Fluid Mechanics, 788, 576-601.

[12] Wang, L. P., Peng, C., Guo, Z., \& Yu, Z. (2016). Lattice Boltzmann simulation of particle-laden turbulent channel flow. Computers \& Fluids, 124, 226-236.

[13] Xu, A., Zhao, T. S., Shi, L., \& Yan, X. H. (2016). Three-dimensional lattice Boltzmann simulation of suspensions containing both micro-and nanoparticles. International Journal of Heat and Fluid Flow, 62, 560-567.

[14] Yu, D., Mei, R., Luo, L. S., \& Shyy, W. (2003). Viscous flow computations with the method of lattice Boltzmann equation. Progress in Aerospace Sciences, 39(5), 329-367.

[15] Wang, L., Guo, Z., Shi, B., \& Zheng, C. (2013). Evaluation of three lattice Boltzmann models for particulate flows. Communications in Computational Physics, 13(04), 1151-1172.

[16] Lorenz, E., Caiazzo, A., \& Hoekstra, A. G. (2009). Corrected momentum exchange method for lattice Boltzmann simulations of suspension flow.Physical Review E, 79(3), 036705.

[17] Ke, C. H., Shu, S., Zhang, H., \& Yuan, H. Z. (2016). LBM-IBM-DEM modelling of magnetic particles in a fluid. Powder Technology.

[18] Shi, X., \& Phan-Thien, N. (2005). Distributed Lagrange multiplier/fictitious domain method in the framework of lattice Boltzmann method for fluid-structure interactions. Journal of Computational Physics, 206(1), 81-94.

[19] Ten Cate, A., Nieuwstad, C. H., Derksen, J. J., \& Van den Akker, H. E. A. (2002). Particle imaging velocimetry experiments and lattice-Boltzmann simulations on a single sphere settling under gravity. Physics of Fluids, 14(11), 4012-4025.

[20] Horowitz, M., \& Williamson, C. H. K. (2010). The effect of Reynolds number on the dynamics and wakes of freely rising and falling spheres. Journal of Fluid Mechanics, 651, 251-294.

[21] Zhou, W., \& Dušek, J. (2015). Chaotic states and order in the chaos of the paths of freely falling and ascending spheres. International Journal of Multiphase Flow, 75, 205-223.

[22] Ern, P., Risso, F., Fabre, D., \& Magnaudet, J. (2012). Wake-induced oscillatory paths of bodies freely rising or falling in fluids. Annual Review of Fluid Mechanics, 44, 97-121.

[23] Fortes, A. F., Joseph, D. D., \& Lundgren, T. S. (1987). Nonlinear mechanics of fluidization of beds of spherical particles. Journal of Fluid Mechanics, 177, 467-483.

[24] Apte, S. V., Martin, M., \& Patankar, N. A. (2009). A numerical method for fully resolved simulation (FRS) of 
rigid particle-flow interactions in complex flows. Journal of Computational Physics, 228(8), 2712-2738.

[25] Yang, J., \& Stern, F. (2015). A non-iterative direct forcing immersed boundary method for strongly-coupled fluid-solid interactions. Journal of Computational Physics, 295, 779-804.

[26] Liao, C. C., Hsiao, W. W., Lin, T. Y., \& Lin, C. A. (2015). Simulations of two sedimenting-interacting sphereswith different sizes and initial configurations using immersed boundary method. Computational Mechanics, 55(6), 1191-1200.

[27] Ernst, M., Dietzel, M., \& Sommerfeld, M. (2013). A lattice Boltzmann method for simulating transport and agglomeration of resolved particles. Acta Mechanica, 224(10), 2425-2449.

[28] Nguyen, N. Q., \& Ladd, A. J. (2005). Sedimentation of hard-sphere suspensions at low Reynolds number. Journal of Fluid Mechanics, 525, 73-104.

[29] Uhlmann, M., \& Doychev, T. (2014). Sedimentation of a dilute suspension of rigid spheres at intermediate Galileo numbers: the effect of clustering upon the particle motion. Journal of Fluid Mechanics, 752, 310-348.

[30] Rothstein, J. P. (2010). Slip on superhydrophobic surfaces. Annual Review of Fluid Mechanics, 42, 89-109.

[31] Sahraoui, M., \& Kaviany, M. (1992). Slip and no-slip velocity boundary conditions at interface of porous, plain media. International Journal of Heat and Mass Transfer, 35(4), 927-943.

[32] Lockerby, D. A., Reese, J. M., Emerson, D. R., \& Barber, R. W. (2004). Velocity boundary condition at solid walls in rarefied gas calculations.Physical Review E, 70(1), 017303.

[33] Navier, C. L. M. H. (1823). Mémoiresur les lois du mouvement des fluides.Mémoires de l'Académie Royale des Sciences de l'Institut de France, 6, 389-440.

[34] Basset, A. B. (1888). A treatise on hydrodynamics. Cambridge: Cambridge University Press.

[35] Keh, H. J., \& Shiau, S. C. (2000). Effects of inertia on the slow motion of aerosol particles. Chemical engineering science, 55(20), 4415-4421.

[36] Feng, Z. G., Michaelides, E. E., \& Mao, S. (2012). On the drag force of a viscous sphere with interfacial slip at small but finite Reynolds numbers.Fluid Dynamics Research, 44(2), 025502.

[37] Luo, H., \& Pozrikidis, C. (2008). Effect of surface slip on Stokes flow past a spherical particle in infinite fluid and near a plane wall. Journal of Engineering Mathematics, 62(1), 1-21.

[38] Chen, S. H., \& Keh, H. J. (1995). Axisymmetric motion of two spherical particles with slip surfaces. Journal of colloid and interface science, 171(1), 63-72.

[39] Li, D., Li, S., Xue, Y., Yang, Y., Su, W., Xia, Z., ... \&Duan, H. (2014).The effect of slip distribution on flow past a circular cylinder. Journal of Fluids and Structures, 51, 211-224.

[40] Mastrokalos, M. E., Papadopoulos, C. I., \& Kaiktsis, L. (2015). Optimal stabilization of a flow past a partially hydrophobic circular cylinder.Computers \& Fluids, 107, 256-271.

[41] Daniello, R., Muralidhar, P., Carron, N., Greene, M., \& Rothstein, J. P. (2013). Influence of slip on vortex-induced motion of a superhydrophobic cylinder. Journal of Fluids and Structures, 42, 358-368.

[42] Van Rij, J., Harman, T., \& Ameel, T. (2012). Slip flow fluid-structure-interaction. International Journal of Thermal Sciences, 58, 9-19.

[43] Mandal, S., Bandopadhyay, A., \& Chakraborty, S. (2015). Effect of interfacial slip on the cross-stream migration of a drop in an unbounded Poiseuille flow. Physical Review E, 92(2), 023002.

[44] Luo, H., \& Pozrikidis, C. (2007). Interception of two spheres with slip surfaces in linear Stokes flow. Journal of Fluid Mechanics, 581, 129-156.

[45] Sun, Q., Klaseboer, E., Khoo, B. C., \& Chan, D. Y. (2013). Stokesian dynamics of pill-shaped Janus particles with stick and slip boundary conditions. Physical Review E, 87(4), 043009.

[46] Ramachandran, A., \& Khair, A. S. (2009). The dynamics and rheology of a dilute suspension of hydrodynamically Janus spheres in a linear flow.Journal of Fluid Mechanics, 633, 233-269. 
[47] Trofa, M., D’Avino, G., Hulsen, M. A., Greco, F., \& Maffettone, P. L. (2016). Numerical simulations of the dynamics of a slippery particle in Newtonian and viscoelastic fluids subjected to shear and Poiseuille flows. Journal of Non-Newtonian Fluid Mechanics, 228, 46-54.

[48] Ginzburg, I., \& d'Humières, D. (2003). Multireflection boundary conditions for lattice Boltzmann models. Physical Review E, 68(6), 066614.

[49] Qian, Y. H., d'Humières, D., \& Lallemand, P. (1992). Lattice BGK models for Navier-Stokes equation. Euro physics Letters, 17(6), 479.

[50] Verhaeghe, F., Luo, L. S., \& Blanpain, B. (2009). Lattice Boltzmann modeling of microchannel flow in slip flow regime. Journal of Computational Physics, 228(1), 147-157.

[51] Tao, S., \& Guo, Z. (2015). Boundary condition for lattice Boltzmann modeling of microscale gas flows with curved walls in the slip regime. Physical Review E, 91(4), 043305.

[52] Rong, L. W., Zhou, Z. Y., \& Yu, A. B. (2015). Lattice-Boltzmann simulation of fluid flow through packed beds of uniform ellipsoids. Powder Technology, 285, 146-156.

[53] Tretheway, D. C., \& Meinhart, C. D. (2002). Apparent fluid slip at hydrophobic microchannel walls. Physics of Fluids, 14(3), L9-L12.

[54] Rauschenberger, P., \& Weigand, B. (2015). Direct numerical simulation of rigid bodies in multiphase flow within anEulerian framework. Journal of Computational Physics, 291, 238-253.

[55] Basset, A. B. (1888). On the motion of a sphere in a viscous liquid.Philosophical Transactions of the Royal Society of London. A, 179, 43-63.

[56] Happel J., Brenner H. (1973). Low Reynolds Number Hydrodynamics. Noordhoff International Publishing: Leiden.

[57] Yuan, W., Deng, J., Cao, Z., \& Mei, M. (2016). Dynamic of one and two elliptical particles settling in oscillatory flow: Period bifurcation and resonance state. Powder Technology, 304, 8-19.

[58] Wang, L., Guo, Z. L., \& Mi, J. C. (2014). Drafting, kissing and tumbling process of two particles with different sizes. Computers \& Fluids, 96, 20-34.

[59] Yang, C., Li, D., \& Masliyah, J. H. (1998). Modeling forced liquid convection in rectangular microchannels with electrokinetic effects. International Journal of Heat and Mass Transfer, 41(24), 4229-4249.

[60] Luo, K., Wei, A., Wang, Z., \& Fan, J. (2013). Fully-resolved DNS study of rotation behaviors of one and two particles settling near a vertical wall. Powder technology, 245, 115-125.

[61] Tao, S., Hu, J. J., \& Guo, Z. L., (2016). An investigation on momentum exchange methods and refilling algorithms for lattice Boltzmann simulation of particulate flows. Computers \& Fluids, 133, 1-14.

[62] Guo, Z. L., Zheng, C. G., \& Shi, B. C. (2002). Non-equilibrium extrapolation method for velocity and pressure boundary conditions in the lattice Boltzmann method. Chinese Physics, 11(4), 366. 


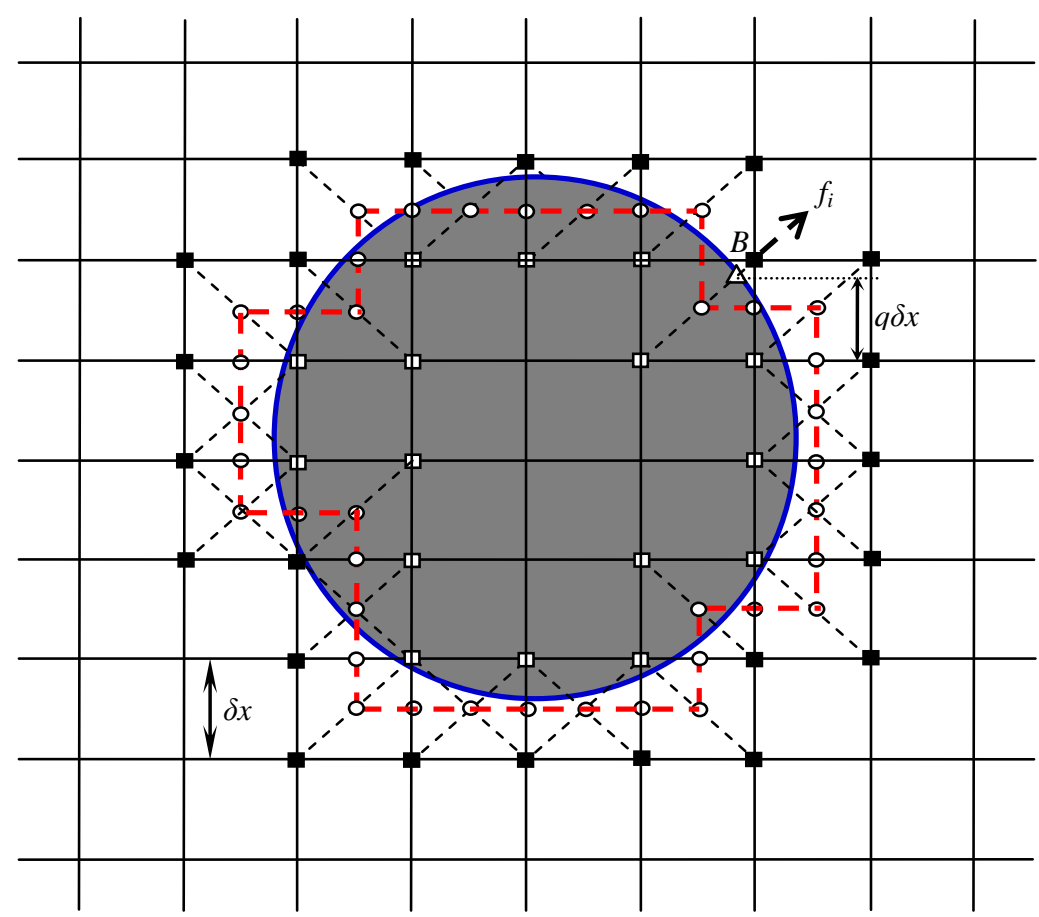

Fig. 1. Schematic of a particle boundary (the solid curve). Open square, boundary node in solid with at least a link intersected by the particle surface;Filled square, boundary node in fluid with at least a link intersected by the surface; Open circle, mid-point of the link of boundary nodes in fluid and solid; Open triangle, intersection point between the link and the wall. The DBB scheme is applied at the filled squares (e.g., point $B$ ).

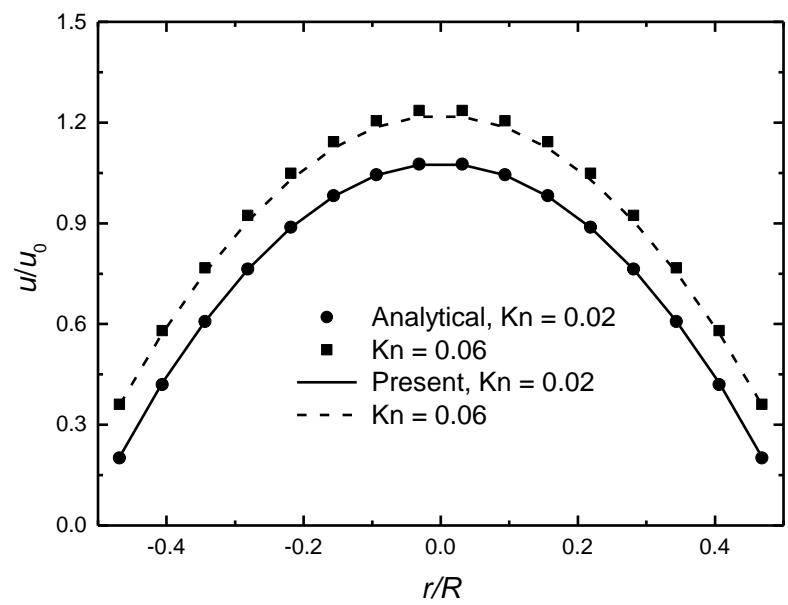

Fig. 2. Velocity profiles of the flow in a cylindrical channel at $\mathrm{Kn}=0.02$ and 0.06 . Symbols: analytical solutions; lines: present LBM results. 


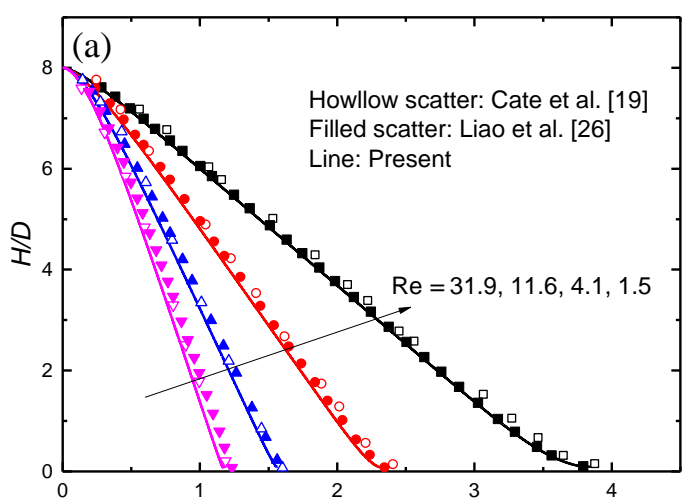

$t(s)$

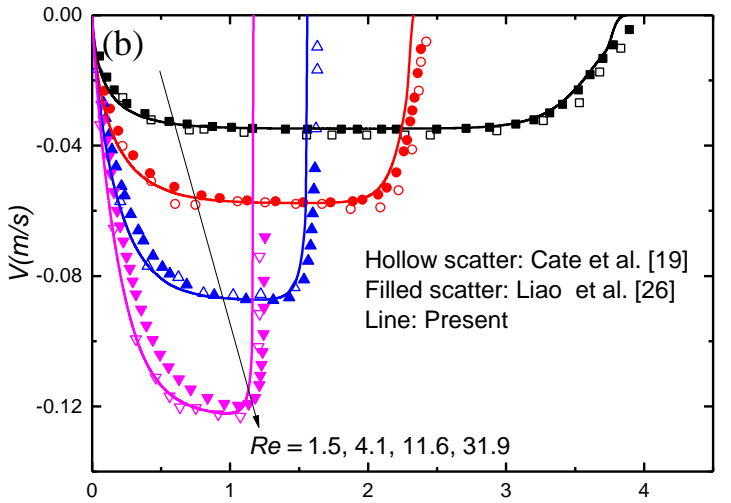

$t(s)$

Fig. 3. Time histories of the vertical gap to the channel bottom and velocity for a sphere settling under gravity in a closed box: (a) normalized gap height $H / D$; (b) settling velocity $V$. $\operatorname{Re}=1.5,4.1,11.6$ and 31.9 correspond to the cases with $\left(\rho_{f}, \mu\right)=\left(0.97 \mathrm{~g} / \mathrm{cm}^{3}, 3.73 \mathrm{~g} / \mathrm{cm} \cdot \mathrm{s}\right),\left(0.965 \mathrm{~g} / \mathrm{cm}^{3}, 2.12 \mathrm{~g} / \mathrm{cm} \cdot \mathrm{s}\right),\left(0.962 \mathrm{~g} / \mathrm{cm}^{3}, 1.13 \mathrm{~g} / \mathrm{cm} \cdot \mathrm{s}\right)$ and $(0.96$ $\left.\mathrm{g} / \mathrm{cm}^{3}, 0.58 \mathrm{~g} / \mathrm{cm} \cdot \mathrm{s}\right)$, respectively.
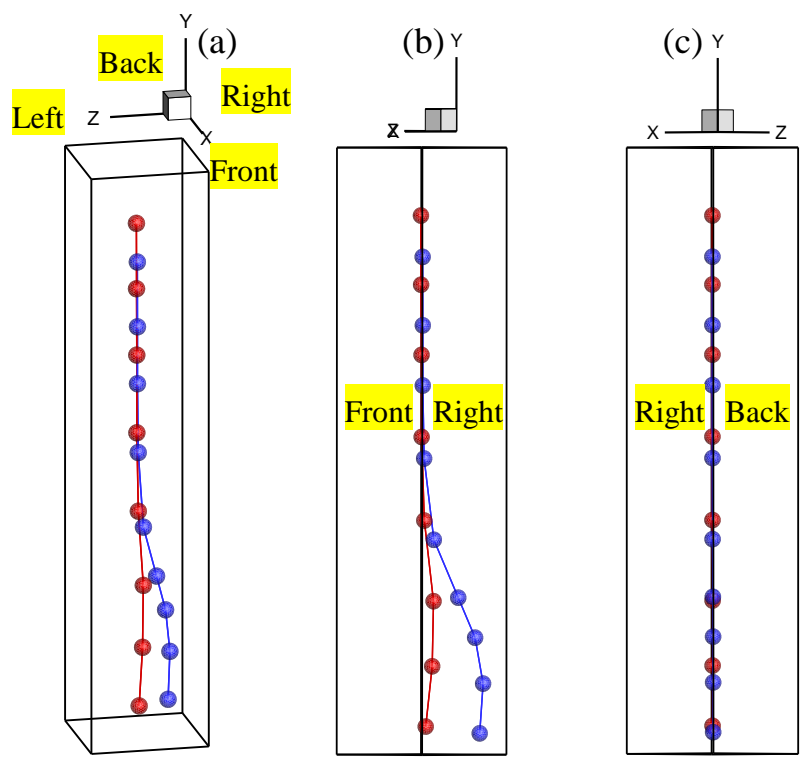

Fig. 4. Instantaneous positions of the two settling spheres undergoing the DKT dynamics. (a) is a 3D view; (b) and (c) are the sectional views obtained by continuously rotating (a) clockwise around the $y$-axis.
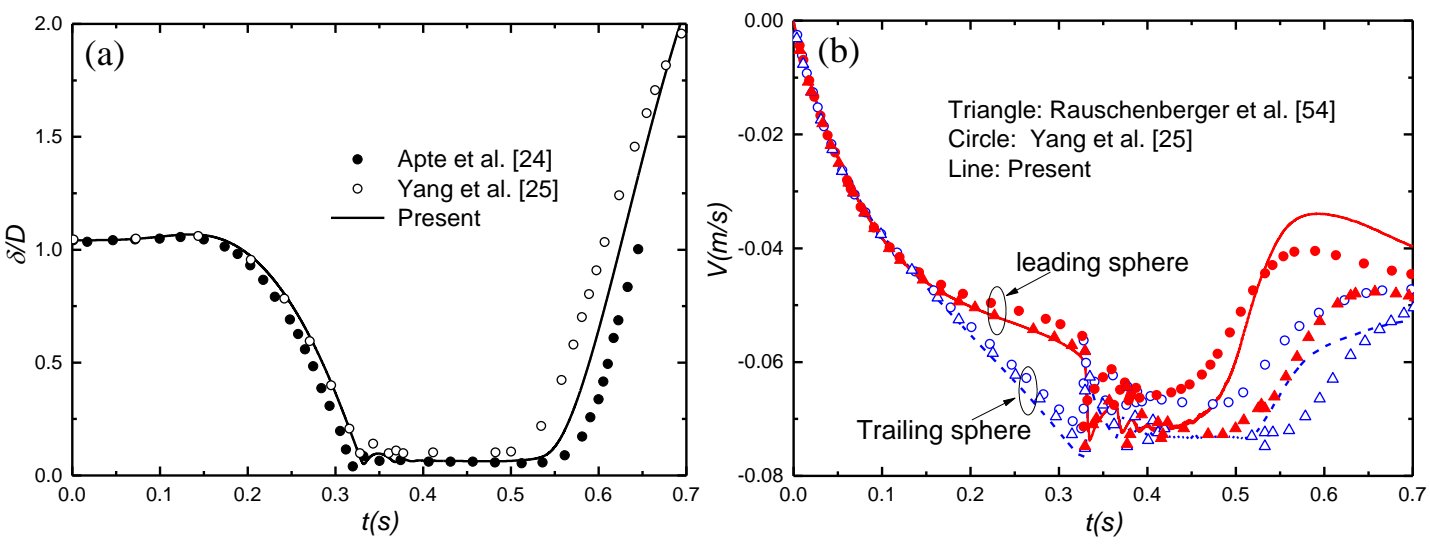

Fig. 5. Time histories of the gap distance between the particles (a) and vertical velocities for the two spheres (b) settling in a quiescent fluid. 


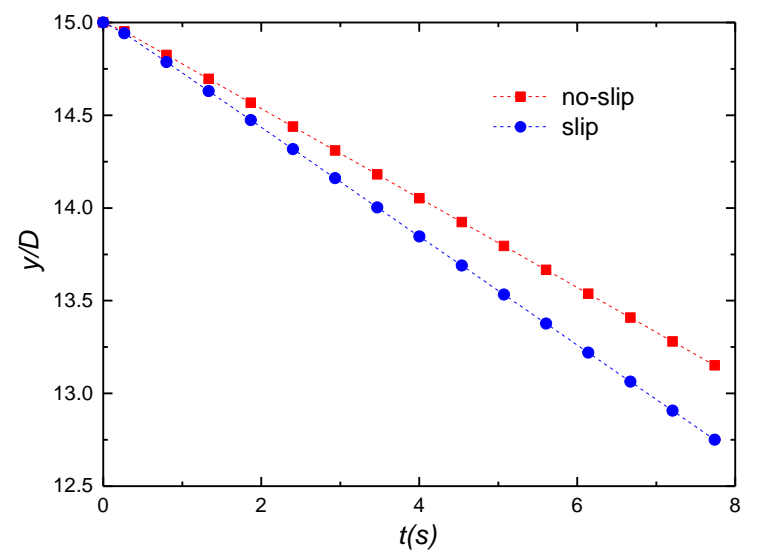

Fig. 6. Instantaneous positions of a sphere settling under gravity with $\rho_{r}=1.001$ and $W^{*}=4$. Square for no-slip particle, and circle for particle with surface slip at $\mathrm{Kn}=0.1$.

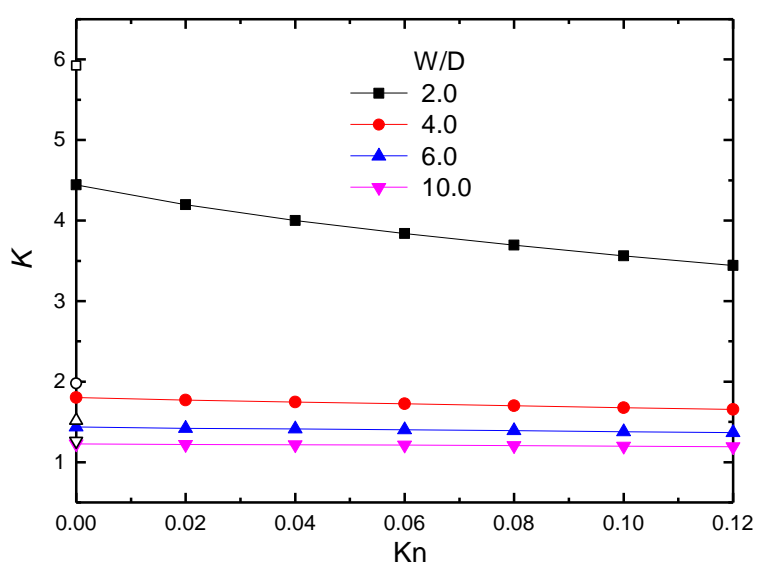

Fig. 7. The wall correction factor $K$ as a function of $K n$ at different blockage ratio $W^{*}$. Open symbols denotes the results of Happle et al. [56] for a no-slip sphere in a tube.

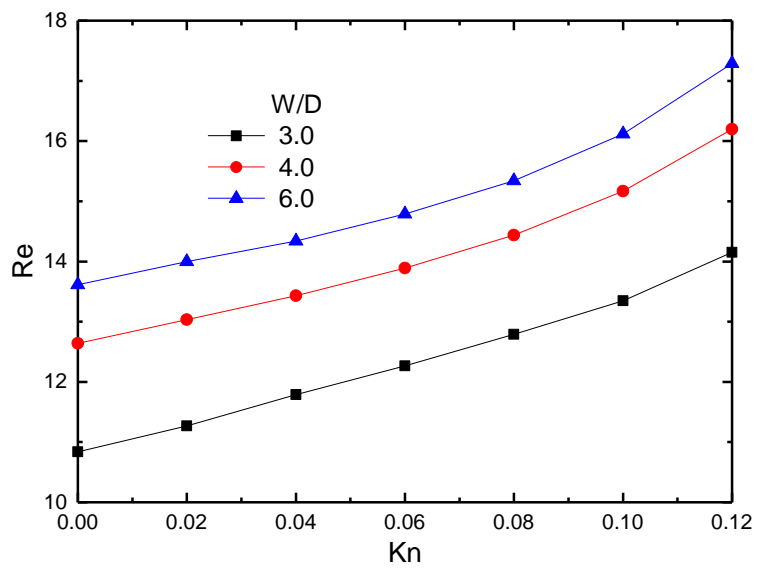

Fig. 8. Particle Reynolds number against Kn with different channel widths at $\rho_{r}=1.1$. 

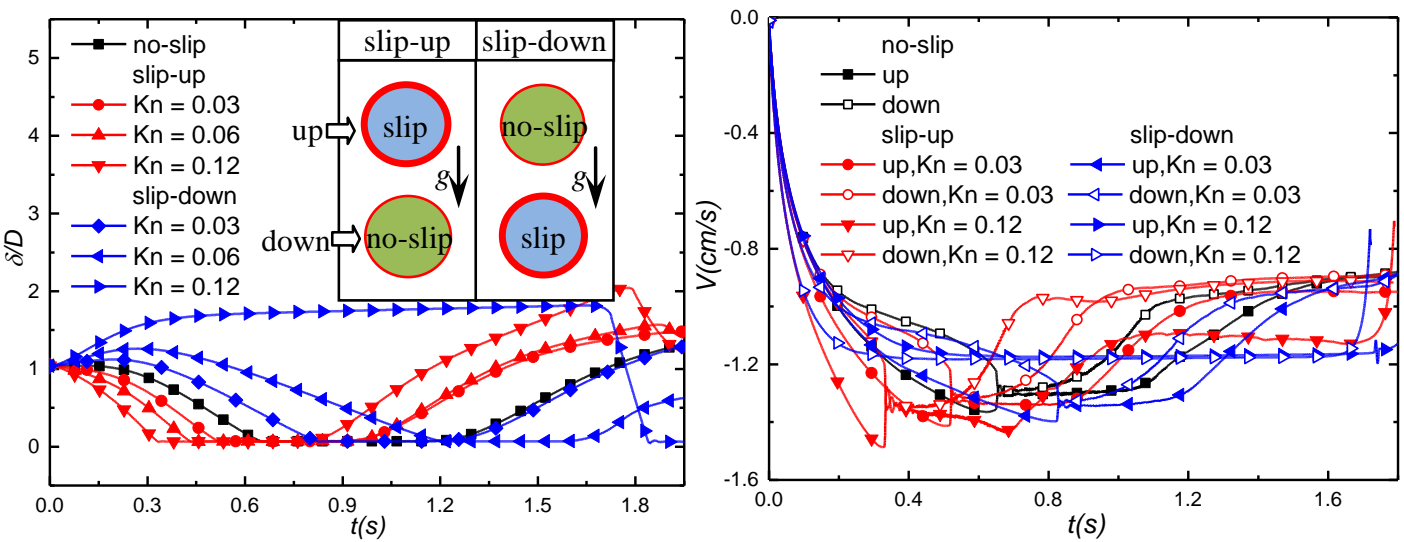

Fig. 9. Time histories of the gap between particles (a) and settling velocities for two spheres (b) in quiescent fluids when only one sphere is slippery.
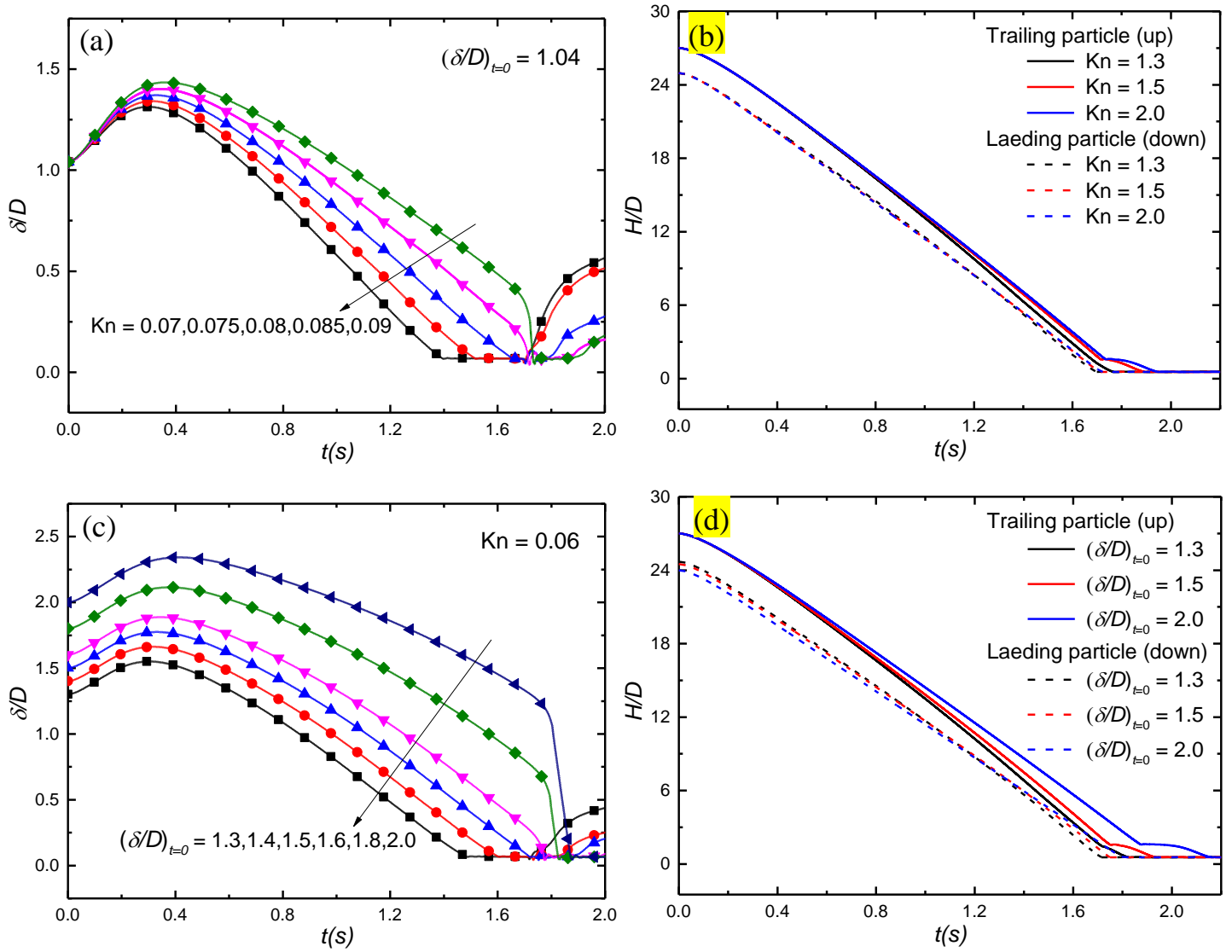

Fig. 10. Time histories of the gap between particles (a, c) and the vertical position of the two spheres (b, d) for the slip-down setup. The initial gap and $\mathrm{Kn}$ are fixed at 1.04 and 0.06 respectively for $(\mathrm{a}, \mathrm{b})$ and (c, d), where the corresponding critical values of $\mathrm{Kn}$ and $\delta / D$ are about 0.085 and 1.5 . 

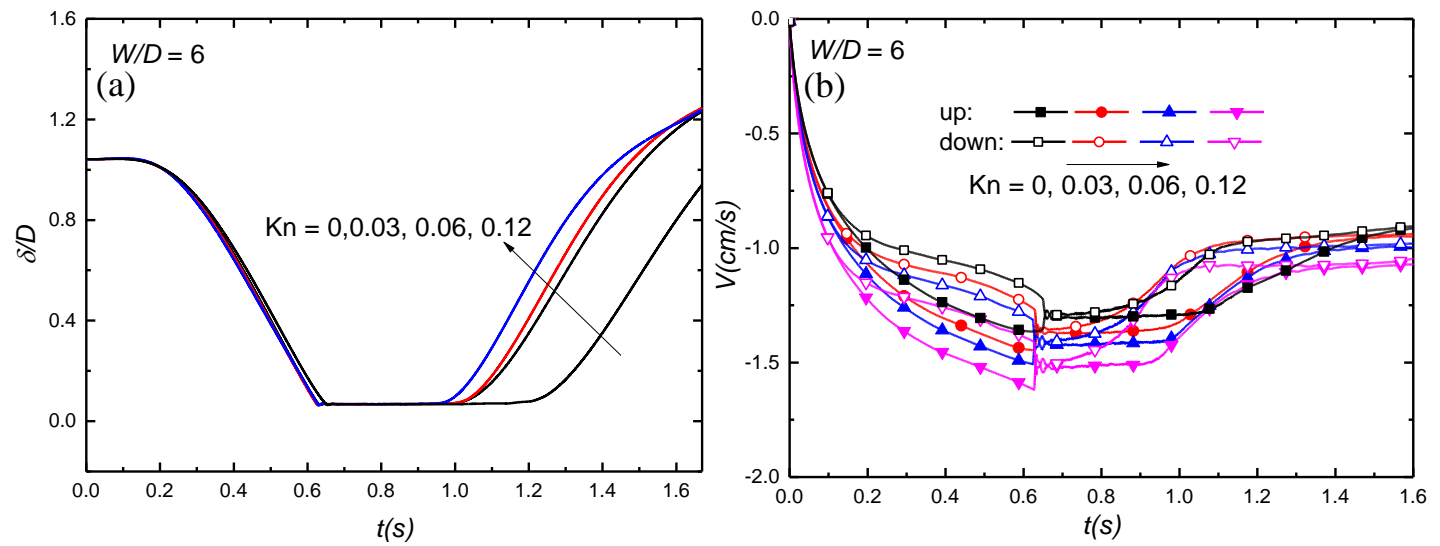

Fig. 11. Time histories of the particle gap (a) and vertical velocity (b) for two slippery spheres in quiescent fluids. Filled scatter, trailing particle; hollow scatter, leading particle.

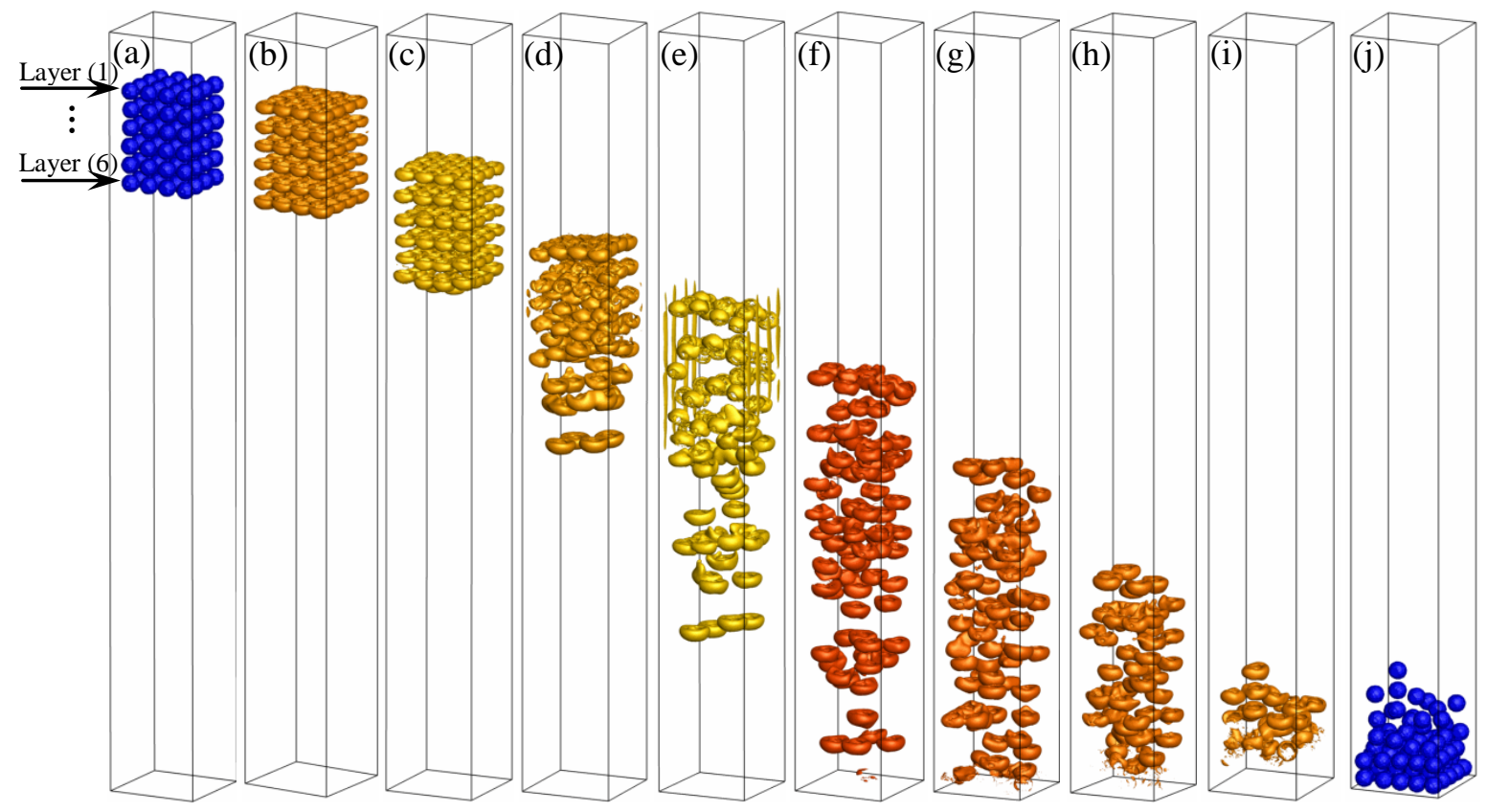

Fig. 12. The sedimentation dynamics of 96 slippery spheres in a container with $\mathrm{Kn}=0.1$. (a): the initial configuration of particles at $t=0$; (b i): Contours of vorticity at times $t=4.8,23.9,47.8,71.6,95.5,119.4,143.3$, $167.1 ;$ (j): the snapshot of particle positions corresponding to (i). 

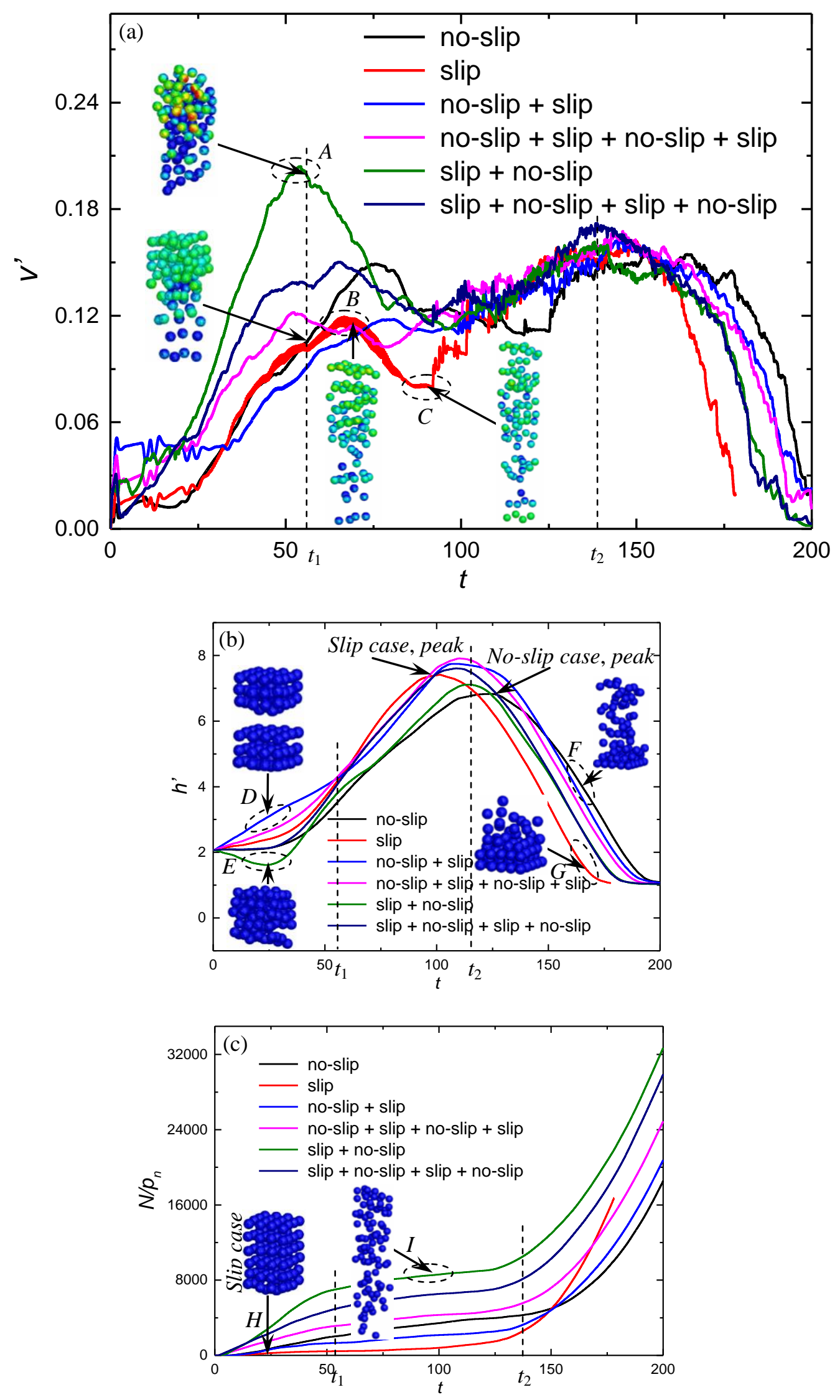

Fig. 13. Time histories of the longitudinal fluctuation velocity $v^{\prime}$, position $h^{\prime}$, and collision number $C N$ for the six configurations. The time is normalized by $T$. 


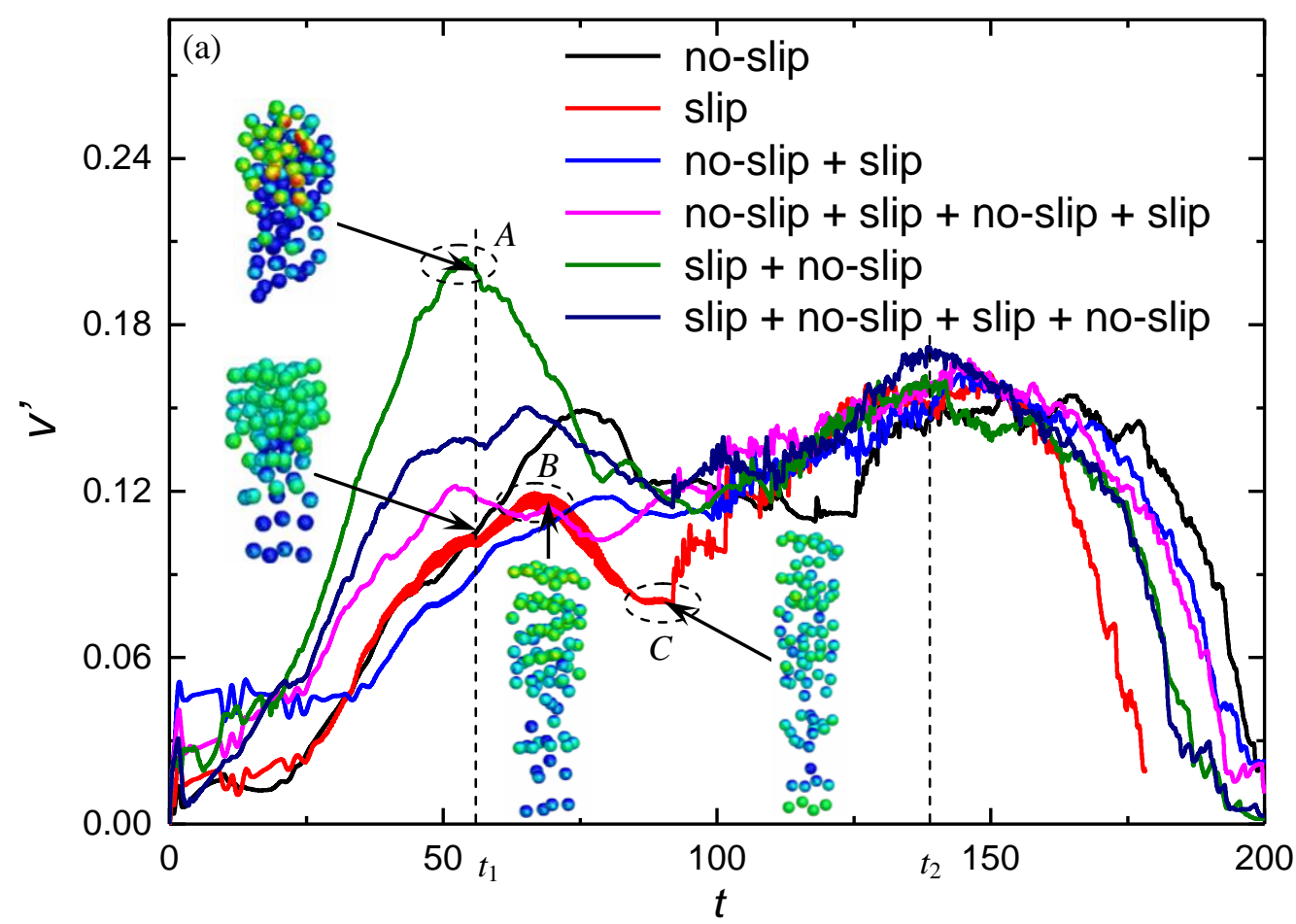

Fig. 13. Time histories of the longitudinal fluctuation velocity $v^{\prime}$ for the six configurations. The time is normalized by $T$. 